\title{
Evidências sobre a abordagem interdisciplinar de equipes de saúde no tratamento da fibromialgia: uma revisão integrativa
}

\author{
Evidence on the interdisciplinary approach of \\ health teams in the treatment of fibromyalgia: an \\ integrative review
}

Como citar este artigo: RESSETTI, JULIANA C; MARTINS, MYLENA F; MÜLLER, ERILDO V.; BORCES, POLLYANNA K. O.; Evidências sobre a abordagem interdisciplinar de equipes de saúde no tratamento da fibromialgia: uma revisão integrativa. Revista Saúde (Sta. Maria). 2020; 46 (1).

\author{
Autor correspondente: \\ Nome: Juliana Campos Ressetti \\ E-mail: jressetti@outlook.com \\ Telefone: (42) 99928-6232 \\ Formação Profissional: Mestranda \\ em Ciências da Saúde pela \\ Universidade Estadual de Ponta \\ Grossa (UEPG) que fica na \\ cidade de Ponta Grossa, Paraná, \\ Brasil. \\ Filiação Institucional: Universidade \\ Estadual de Ponta Grossa (UEPG) \\ Endereço para correspondencia: \\ Rua: Prof. Jugurta Conçalves de \\ Oliveira $n^{\circ}: 237$ \\ Bairro: Contorno \\ Cidade: Ponta Grossa \\ Estado: Paraná \\ CEP: 84052-460
}

Data de Submissão:

31/08/2018

Data de aceite:

16/01/2020

Conflito de Interesse: Não há conflito de interesse

\section{(cc) $\mathrm{BY}-\mathrm{NC}-\mathrm{ND}$}

\author{
Juliana Campos Ressetti, Mylena de França Martins, Erildo Vicente Müller, \\ Pollyanna Kássia de Oliveira Borges
}

\section{RESUMO}

A fibromialgia é considerada uma síndrome de dor crônica, sendo caracterizada pela manifestação dolorosa difusa no sistema músculo-esquelético, que pode estar associada a outros sintomas. Devido à complexidade da fibromialgia, o diagnóstico e manejo são desafiadores para os profissionais da saúde. Deste modo, é necessário que sejam realizadas ações que contemplem o atendimento integral pelas equipes multiprofissionais com a abordagem interdisciplinar. Frente a isso, foi realizada uma revisão integrativa da literatura com o objetivo de identificar as evidências sobre a atuação das equipes interdisciplinares de saúde para o tratamento de pacientes com fibromialgia, por meio dos estudos publicados na literatura de 2008 a 2018. Nos resultados, foram identificados os países de origem dos estudos, 0 delineamento das pesquisas e as diferentes categorias profissionais envolvidas nos cuidados, como os profissionais da área da medicina, fisioterapia, psicologia, terapia ocupacional, enfermagem, assistência social, fonoaudiologia, nutrição, educação física e mestrando de enfermagem. Foram encontrados diferentes resultados nos estudos analisados, sendo observado melhora de indicadores da qualidade de vida, aumento de capacidade funcional, redução do nível da dor e de outros sintomas característicos da síndrome. Foi observado também a escassez de estudos interdisciplinares referente à fibromialgia e confusão entre o tema multidisciplinar e interdisciplinar. Conclui-se que a atuação das equipes interdisciplinares tem um retorno positivo do paciente por meio dos tratamentos realizados encontrados na literatura, sendo viáveis novos estudos de abordagem interdisciplinar para o tratamento do paciente com a fibromialgia.

PALAVRAS-CHAVE: Práticas interdisciplinares; Dor crônica; Profissionais da saúde; Fibromialgia.

\section{ABSTRACT}

Fibromyalgia is considered a chronic pain syndrome and is characterized by diffuse painful manifestation in the musculoskeletal system, which may be associated with other symptoms. Due to the complexity of fibromyalgia, diagnosis and management are challenging for health professionals. Thus, it is necessary to perform actions that contemplate comprehensive care by multiprofessional teams with an interdisciplinary approach. In view of this, an integrative review of literature was performed to identify the evidence on the role of interdisciplinary health teams in the treatment of patients with fibromyalgia through studies published in the literature from 2008 to 2018. In the results, the countries of origin of the studies were identified. the delineation of research and the different professional categories involved in care, such as professionals in the fields of medicine, physiotherapy, psychology, occupational therapy, nursing, social assistance, speech therapy, nutrition, physical education and nursing master's student. Different results were found in the analyzed studies, being observed improvement of quality of life indicators, increase of functional capacity, reduction of pain level and other characteristic symptoms of the syndrome. There was also a shortage of interdisciplinary studies regarding fibromyalgia and confusion between the multidisciplinary and interdisciplinary theme. It is concluded that the performance of interdisciplinary teams has a positive return of the patient through the treatments found in the literature, being feasible new studies of interdisciplinary approach to the treatment of patients with fibromyalgia. 


\section{INTRODUÇÃO}

A fibromialgia é considerada uma síndrome de dor crônica que acomete principalmente o público feminino e é caracterizada pela manifestação dolorosa difusa no sistema músculo-esquelético, podendo estar associada a sintomas de diferentes sistemas, como o distúrbio de sono, rigidez matinal, alteração na cognição, fadiga e doenças psicossociais ${ }^{1,2,3}$.

A fibromialgia tem prevalência de $0,66 \%$ a $4,40 \%$ na população mundial, sendo responsável por $15 \%$ das consultas ambulatoriais de reumatologia e 5\% a 10\% nos ambulatórios de clínica geral. No Brasil, a síndrome acomete cerca de $2 \%$ da população ${ }^{4}$. Devido à complexidade das comorbidades envolvidas nesta síndrome, o seu diagnóstico e manejo surgem como um desafio para os profissionais da saúde. Deste modo, é crucial desenvolver ações que disponibilizem condições favoráveis para a reabilitação de pessoas que apresentem a fibromialgia.

O tratamento da fibromialgia pode ser realizado de maneira medicamentosa e por abordagens não medicamentosas, em consonância com a necessidade e intensidade dos sintomas do paciente ${ }^{5}$. De acordo com as recomendações revisadas no ano de 2015 para o tratamento da fibromialgia concebidos pela EULAR (Liga Europeia contra o Reumatismo), o tratamento não medicamentoso é proposto como terapia de primeira linha, e na ausência da melhora do paciente, o tratamento medicamentoso pode ser incluído à terapêutica ${ }^{6}$.

No que se refere a abordagem das equipes de saúde, pode ser apontado a atuação de equipes multiprofissionais com a atuação multidisciplinar, que em resumo, resulta de uma interação entre os envolvidos no cotidiano de suas práticas na oferta do cuidado de saúde, e reúnem no mesmo espaço, diferentes perspectivas e interesses ${ }^{7}$, desta maneira, implicando em uma justaposição de disciplinas ${ }^{8}$. Não obstante, está interação não necessariamente poderá resultar em trabalho realizado de modo coordenado e em equipe ${ }^{8}$.

Em contrapartida, a abordagem interdisciplinar se diferencia da multidisciplinar, pois segundo Scherer, Pires e $\operatorname{Jean}^{9}$, a interdisciplinaridade "exige a integração não somente de saberes, mas também de práticas, e integra e renormaliza as disciplinas e as profissões delas decorrentes, concretizando, ao final, a íntima relação entre conhecimento e ação". Ainda, Costa ${ }^{8}$ acrescenta que existe a "necessidade de reformulação do discurso e da práxis da saúde pública. Essa reconstrução deve se dar dentro de uma lógica interdisciplinar, pois só assim será possível retomar a complexidade do objeto da saúde". Deste modo, se faz necessário o reconhecimento das práticas interdisciplinares no tratamento desta síndrome que é caracterizada por múltiplos sintomas e que necessita da atenção integral oferecida pelos profissionais de saúde.

Diante desses pressupostos, a presente revisão integrativa teve como o objetivo identificar as evidências sobre a atuação das equipes interdisciplinares de saúde para o tratamento de pacientes com fibromialgia, por meio dos estudos publicados na literatura de 2008 a 2018. 


\section{MATERIAL E MÉTODO}

A fim de responder o objetivo proposto no presente estudo, foi realizada uma revisão integrativa. Este método de pesquisa permite a integralização de evidências de estudos de diferentes delineamentos sobre determinado tema, o qual pode contribuir para a construção de uma compreensão abrangente sobre o assunto ${ }^{10}$. A execução da coleta de dados se deu no período de junho a agosto do ano de 2018.

Devida à existência das diferentes disfunções que envolvem a fibromialgia, o cuidado por meio de ações de equipes multimodais de saúde é uma das alternativas de tratamento. Desta forma, a questão norteadora da presente revisão é: - Quais são os benefícios de tratamentos de equipes interdisciplinares de saúde nos cuidados da fibromialgia?

O levantamento de artigos foi realizado por meio de busca nas bases de dados Medical Literature Analysis and Retrieval System Online (MEDLINE), Literatura Latino-Americana e do Caribe em Ciências da Saúde (LILACS), Índice Bibliográfico Español en Ciencias de la Salud (IBECS) e Scientific Electronic Library Online (SciELO). Para o rastreio das pesquisas foi utilizada a combinação dos termos fibromialgia (fibromyalgia) e equipe interdisciplinar (interdisciplinary team); fibromialgia (fibromyalgia) e interdisciplinar (interdisciplinary) nos idiomas português e inglês, sendo empregado 0 operador booleano "AND" entre as palavras.

Os critérios de inclusão adotados foram: estudos no formato de artigos, publicados nos anos de 2008 a 2018 com a temática sobre a abordagem interdisciplinar no cuidado de pacientes com fibromialgia nos idiomas inglês e português. Já os critérios de exclusão foram: estudos declarados com o caráter multidisciplinar; estudos com ênfase em uma determinada área dentro da equipe interdisciplinar; relatórios de comitês de especialidades; revisão sistemática e metanálise.

O processo de seleção dos artigos foi guiado na sequência de três etapas. A primeira etapa de seleção foi realizada por meio da leitura dos títulos das pesquisas, a segunda etapa foi efetuada a seleção pela leitura do resumo e a terceira etapa se deu pela leitura do estudo na íntegra. Na sequência, está disposto um fluxograma do processo de seleção dos artigos revisados (Figura 1).

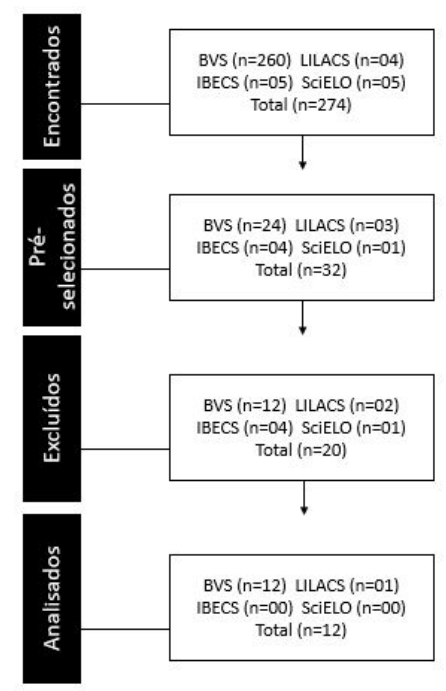

Figura 1 - Fluxograma do processo de seleção dos artigos revisados de acordo com a base de dados. Legenda: Primeiramente foram 
encontrados respectivos artigos na busca em cada base de dados: 260 artigos na MEDLINE, 4 artigos na LILACS, 5 artigos no IBECS, 5 artigos na SciELO, totalizando 274 artigos. Destes artigos encontrados, foram pré-selecionados em cada base de dados: 24 artigos na MEDLINE, 3 artigos na LILACS, 4 artigos no IBECS, 1 artigo na SciELO, totalizando 32 artigos. Após a pré-seleção foram realizadas exclusões de artigos que não se enquadravam nos critérios de inclusão em cada base de dados: 12 artigos na MEDLINE, 2 artigos na LILACS, 4 artigos no IBECS, 1 artigo na SciELO, totalizando 19 artigos. Por fim, foram estabelecidos os artigos para a analisada em cada base de dados: 12 artigos MEDLINE, nenhum artigo LILACS, nenhum artigo IBECS, nenhum artigo SciELO, totalizando 12 artigos.

Para sistematizar as informações provenientes dos estudos coletos, foram elaborados dois quadros contendo os itens: autor (ano de publicação), local do estudo, objetivo, delineamento da pesquisa, atores envolvidos na ação interdisciplinar, métodos interdisciplinares empregados e principais achados. Após a leitura na integra dos artigos selecionados, estes foram organizados e analisados de acordo com os itens supracitados.

Para elucidação dos principais resultados das pesquisas analisadas, foi organizado três agrupamentos, que foram distribuídos de acordo com os objetivos dos artigos analisados. No grupo A, estão alocados os estudos que avaliaram os benefícios promovidos pelos programas de tratamento interdisciplinar. No grupo $B$, estão agrupados os estudos que tiveram como objeto identificar os preditores que poderiam estar relacionados com a responsividade do paciente ao tratamento interdisciplinar, e por fim, no grupo $C$ encontram-se os estudos em que avaliaram os efeitos do tratamento com o manejo interdisciplinar após o período da intervenção a longo prazo.

O presente estudo foi construído na disciplina de Estudos Interdisciplinares em Doenças Crônicas do Programa de Mestrado Interdisciplinar em Ciências da Saúde da UEPG como parte do processo formativo e com a finalidade de promover o desenvolvimento da habilidade de buscar recursos teóricos que instrumentalizassem os profissionais de saúde no conhecimento das ferramentas de cuidado interdisciplinar empregados na fibromialgia.

\section{RESULTADO}

Dentre os 12 estudos analisados, 25\% foram publicados em 2016, 16,7\% em 2017, 16,7\% em 2014, 16,7\% em 2012, 8,3\% em 2015, 8,3\% em 2011 e 8,3\% em 2010. Não obstante, houve ausência de estudos nos anos de 2008, 2009, 2013 e 2018 de acordo com os critérios de busca empregados.

As pesquisas foram produzidas em países como Espanha (25\%), Estados Unidos da América (25\%), Brasil (16,7\%), Dinamarca (16,7\%), Canadá (8,3\%) e Turquia (8,3\%), tendo mulheres com a faixa-etária acima de 40 anos como público-alvo de maior número de participação das intervenções experimentais. No que se refere ao delineamento das pesquisas, o ensaio clínico randomizado foi o mais frequente (50\%), seguido de estudo prospectivo de coorte (25\%), ensaio clínico quase-randomizado (8,33\%), pesquisa de satisfação e pesquisa qualitativa participativa $(8,33 \%)$. 
Os estudos visaram essencialmente avaliar o efeito dos programas de tratamento e a identificar os preditores envolvidos na melhora dos pacientes. A seguir, encontra-se um quadro com detalhamento com informações de acordo com o autor, ano de publicação, país de origem, objetivo e o delineamento da pesquisa (Quadro 1).

\begin{tabular}{|c|c|c|c|c|}
\hline $\mathrm{N}^{0}$ & Autor (ano) & Local & Objetivo & $\begin{array}{l}\text { Delineamento da } \\
\text { pesquisa }\end{array}$ \\
\hline 1 & $\begin{array}{l}\text { Bülow C, Amris K, } \\
\text { Bandak E, et al. }{ }^{11} \\
(2017)\end{array}$ & Dinamarca & $\begin{array}{l}\text { Explorar e comparar os resultados dos } \\
\text { programas de adaptação e atividade } \\
\text { física em relação à capacidade de AVD } \\
\text { após a reabilitação interdisciplinar. }\end{array}$ & $\begin{array}{l}\text { Ensaio clínico } \\
\text { quase-randomizado }\end{array}$ \\
\hline 2 & $\begin{array}{l}\text { Martín J, Torre F, } \\
\text { Aguirre U, et al. }{ }^{12} \\
(2017)\end{array}$ & Espanha & $\begin{array}{l}\text { Identificar os preditores do impacto } \\
\text { de longo prazo na QVRS após } \\
\text { uma intervenção interdisciplinar. }\end{array}$ & $\begin{array}{l}\text { Estudo de coorte } \\
\text { prospectivo }\end{array}$ \\
\hline 3 & $\begin{array}{l}\text { Miranda NACG, } \\
\text { Berardinelli LMM, } \\
\text { Sabóia VM, et al. } 1^{3} \\
(2016)\end{array}$ & Brasil & $\begin{array}{l}\text { Analisar o modelo da terapia comunitária } \\
\text { integrativa para o empoderamento dos } \\
\text { pacientes e discutir a repercussão no } \\
\text { processo saúde-doença e autocuidado. }\end{array}$ & $\begin{array}{l}\text { Pesquisa } \\
\text { qualitativa } \\
\text { participativa }\end{array}$ \\
\hline 4 & $\begin{array}{l}\text { Amris K, Luta G, } \\
\text { Christensen R, et al. }{ }^{14} \\
(2016)\end{array}$ & Dinamarca & $\begin{array}{l}\text { Investigar os preditores de melhora } \\
\text { na capacidade observada para } \\
\text { gerenciar as AVD. }\end{array}$ & $\begin{array}{l}\text { Ensaio clínico } \\
\text { randomizado }\end{array}$ \\
\hline 5 & $\begin{array}{l}\text { Saral I, Sindel D, } \\
\text { Esmaeilzadeh S, et al. }{ }^{15} \\
(2016)\end{array}$ & Turquia & $\begin{array}{l}\text { Determinar os efeitos das abordagens } \\
\text { de longo e curto prazo na redução da } \\
\text { dor, fadiga, qualidade do sono, sintomas } \\
\text { depressivos, melhoria da QVRS e de } \\
\text { funções físicas. }\end{array}$ & $\begin{array}{l}\text { Ensaio clínico } \\
\text { randomizado }\end{array}$ \\
\hline 6 & $\begin{array}{l}\text { Bourgault P, Lacasse A, } \\
\text { Marchand S, et al. }{ }^{16} \\
(2015)\end{array}$ & Canadá & $\begin{array}{l}\text { Avaliar, quantitativa e qualitativamente, } \\
\text { a eficácia de uma intervenção } \\
\text { interdisciplinar para o autogerenciamento. }\end{array}$ & $\begin{array}{l}\text { Ensaio clínico } \\
\text { randomizado }\end{array}$ \\
\hline 7 & $\begin{array}{l}\text { Martins MR, Gritti CC, } \\
\text { Santos Jr R, et al. }{ }^{17} \\
(2014)\end{array}$ & Brasil & $\begin{array}{l}\text { Avaliar a eficácia de um programa } \\
\text { interdisciplinar semanal para } \\
\text { determinar a efetividade em } \\
\text { curto e médio prazo. }\end{array}$ & $\begin{array}{l}\text { Ensaio clínico } \\
\text { randomizado }\end{array}$ \\
\hline 8 & $\begin{array}{l}\text { Martín J, Torre F, } \\
\text { Padierna A, et al. }{ }^{18} \\
\text { (2014) }\end{array}$ & Espanha & $\begin{array}{l}\text { Avaliar uma intervenção interdisciplinar } \\
\text { na melhora da QVRS comparado com } \\
\text { resultados do cuidado padrão farmacológico } \\
\text { e identificar preditores de melhoria }\end{array}$ & $\begin{array}{l}\text { Ensaio clínico } \\
\text { randomizado }\end{array}$ \\
\hline 9 & $\begin{array}{l}\text { Martín J, Torre F, } \\
\text { Padierna A, et al. }{ }^{19} \\
\text { (2012) }\end{array}$ & Espanha & $\begin{array}{l}\text { Avaliar as mudanças ao longo do tempo } \\
\text { na saúde biopsicossocial e na QV } \\
\text { da intervenção experimental comparado } \\
\text { com o cuidado habitual. }\end{array}$ & $\begin{array}{l}\text { Ensaio clínico } \\
\text { randomizado }\end{array}$ \\
\hline
\end{tabular}




\begin{tabular}{|c|c|c|c|c|}
\hline 10 & 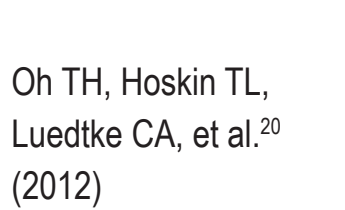 & Estados Unidos & $\begin{array}{l}\text { Determinar quais características do } \\
\text { resposta positiva ao programa } \\
\text { paciente estão associadas à } \\
\text { interdisciplinar breve. }\end{array}$ & $\begin{array}{l}\text { Estudo de coorte } \\
\text { prospectivo }\end{array}$ \\
\hline 11 & $\begin{array}{l}\text { Jones KD, Bennett RM, } \\
\text { Ward RL, et al. }{ }^{21} \\
(2011)\end{array}$ & Estados Unidos & $\begin{array}{l}\text { Avaliar a satisfação do paciente } \\
\text { em uma clínica profissional } \\
\text { de fibromialgia de meio turno. }\end{array}$ & $\begin{array}{l}\text { Pesquisa de } \\
\text { satisfação }\end{array}$ \\
\hline 12 & $\begin{array}{l}\text { Oh TH, Stueve MH, } \\
\text { Hoskin TL, et al. }{ }^{22} \\
(2010)\end{array}$ & Estados Unidos & $\begin{array}{l}\text { Avaliar o impacto e benefício a longo } \\
\text { prazo de um programa de tratamento } \\
\text { interdisciplinar breve de } 11 / 2 \text { dias. }\end{array}$ & $\begin{array}{l}\text { Estudo de coorte } \\
\text { prospectivo }\end{array}$ \\
\hline
\end{tabular}

Quadro 1 - Artigos inclusos na revisão integrativa segundo autor (ano), país de origem, objetivo e o delineamento da pesquisa. QV = qualidade de vida; QVRS= qualidade de vida relacionada à saúde; $A V D$ = atividades de vida diária.

Dos 12 artigos analisados, foram identificadas 11 categorias profissionais que integravam as equipes de intervenção, nas quais foram citadas com a seguinte frequência: 10 medicina $(21,74 \%), 9$ fisioterapia $(19,57 \%), 8$ psicologia (17,39\%), 6 terapia ocupacional (13,04\%), 6 enfermagem (13,04\%), 1 assistência social (2,17\%), 1 fonoaudiologia $(2,17 \%)$, 1 nutrição (2,17\%), 1 profissional de educação física (2,17\%), 1 mestrando de enfermagem (2,17\%) e 2 sem categoria profissional declarada $(4,26 \%)$. As principais atividades empregadas observadas foram as atividades educacionais, nas quais eram voltadas para a orientação e conhecimento da síndrome; a psicoterapia, mais especificamente a realização de técnicas da terapia cognitivo comportamental (TCC); a orientação e execução de exercícios físicos; recomendações de adaptações de atividades de vida diária; diálogos e troca de experiências entre os pacientes; e o uso de tratamento medicamentoso padrão para a fibromialgia. No que se refere ao tempo de duração e frequência das intervenções, houve ampla variedade, em que se identificou principalmente frequência de uma e duas sessões semanais, com a duração do período de intervenção variando de duas, seis e 12 semanas.

Em virtude dos objetivos propostos pelas respectivas pesquisas, foram encontrados resultados diversificados nos estudos analisados. Pode ser observado melhora de indicadores da qualidade de vida (QV), aumento de capacidade funcional, redução do nível da dor, melhora de sintomas de ansiedade e depressão e do impacto da fibromialgia na vida do paciente, além da identificação de preditores para melhora das variáveis avaliadas. Na sequência será exposto um quadro contendo as informações supracitadas dos estudos analisados (Quadro 2). 
Programa de reabilitação interdisciplinar: Médico reumatologista, psicólogo, enfermeiro, terapeuta ocupacional e fisioterapeuta; Programa adicional ativo: fisioterapeuta; Programa adicional adapt.: terapeuta ocupacional.
Duas semanas de um programa de reabilitação interdisciplinar (programa ambulatorial, com duração entre 3/5hrs diárias, que incluiu conferência da equipe sobre a melhora da capacidade funcional e enfrentamento da dor); seguido pela alocação dos pacientes em grupo programa de atividade física (12 semanas) e grupo de adaptação (16 semanas).
Melhora da capacidade de AVD motora e do processo na maioria dos participantes independente da alocação do grupo após a reabilitação interdisciplinar. Os programas adicionais parecem ter melhorado consideravelmente os ganhos funcionais.
Médico, psicólogo clínico e fisioterapeuta.

2

Médico, profissional de educação física, psicólogo, nutricionista, enfermeiro, fonoaudiólogo, e um Mestrando de enfermagem.
Duas sessões coletivas semanais por seis semanas de atividades educacionais, fisioterapêuticas (exercícios) e TCC.
Os preditores envolvidos no impacto da fibromialgia na QV foram o estado civil, número de doenças dor e de concomitantes, anos de convívio da presença de ansiedade.
Uma vez por semana de atividades de educação adaptativa em saúde; uma vez por semana de terapia em grupo e terapia em grupo quinzenal (convivência). No processo foi disponibilizado atendimento nutricional, realização de atividades físicas e atendimento da enfermagem. Cada uma destas etapas teve a duração de três meses, totalizando nove meses de duração.
A estratégia revelou uma alternativa de cuidado de promoção à saúde por seu caráter dialógico e interdisciplinar, possibilitando um espaço integrador que busca o fortalecimento do autocuidado, a descoberta de potenciais como alternativa para discutir os problemas de saúde e reorientação de posicionamentos. Além de uma opção de custo reduzido para a saúde pública e por mostrar que a interdisciplinariedade nesse caso teve benefícios melhores do que se fosse trabalhado por profissionais isolados.
Médico reumatologista, psicólogo, enfermeiro, terapeuta ocupacional e 4 fisioterapeuta.
Duas semanas de programa de reabilitação interdisciplinar com duração entre 3/5hrs diárias, com abordagem participativa e interativa por meio da educação e adaptação sobre o enfrentamento da dor e o aumento da capacidade das AVD.
Os pacientes mais propensos a alcançar melhora clinicamente significava na capacidade funcional eram aqueles que tiveram baixa ingestão de analgésicos, elevado nível atual de dor e pontuação total no questionário de detecção de dor.

\begin{abstract}
Pesquisadores sem a área de atuação declarada e psicólogo.
\end{abstract}

Grupo de longo prazo: 10 sessões de TCC (uma sessão semanal de $3 \mathrm{~h}$ durante 10 semanas), treinamento físico (um dia inteiro) e atividades educacionais (um dia inteiro). Grupo de curto prazo: atividades educacionais, treinamento físico e um breve programa de TCC ao longo de dois dias consecutivos.
Ambas as abordagens foram eficazes para reduzir a intensidade da dor e 0 número de tender points, aumentar 0 limiar de dor à pressão, controlar a atividade da doença e melhorar o status também foi eficaz ao reduzir a gravidade da fadiga e ao melhorar os componentes físicos da QVRS.

$6 \quad \begin{aligned} & \text { Área de atuação não } \\ & \text { declarada. Dois facilitadores } \\ & \text { encarregados ao aspecto }\end{aligned}$

Nove sessões com duração de 2,5h, distribuídos em grupos de 8 pacientes, sendo as principais atividades envolvidas
Melhora da impressão global de dor, de funcionamento e de $\mathrm{QV}$, bem como em aumento da percepção 
psicológico e aspecto físico.

Paciente designado como

"especialista" de sua condição. foram ferramentas psicoeducacionais, TCC e os exercícios adaptados. Uma sessão foi designada ao tratamento farmacológico e não farmacológico. do alívio da dor.

Aumento da capacidade funcional e motivação, melhora do controle do sono, da ansiedade e da depressão.
Médico, terapeuta ocupacional, fisioterapeuta, psicólogo e assistente social.
Sessões semanais com duração de $1 \mathrm{~h}$ em 12 semanas de atividades como exercícios de fortalecimento, aptidão cardiorrespiratória, alongamentos e ergométricos, técnicas de relaxamento e programas educativos, psicossociais e ocupacionais. Houve orientação de realização de exercícios domiciliares.
Médico, psicólogo clínico e fisioterapeuta.
Duas sessões semanais em seis semanas, metade das sessões foram de $1 \mathrm{~h}$ com o psicólogo e 45min de atividades educativas com o médico e psicólogo e a outra metade de $1 \mathrm{~h}$ com 8 o psicólogo e 45min com o fisioterapeuta. As atividades desenvolvidas foram educacionais, TCC e treinamento físico progressivo. receberam tratamento farmacológico padrão. O grupo controle e o grupo experimental
Melhora da capacidade funcional, nível de dor, e escore geral da QVRS e o número de doenças foi identificado como preditor dessa melhora. 0 tratamento interdisciplinar produziu melhores resultados quando comparado à farmacoterapia padrão. Médico, psicólogo clínico e fisioterapeuta.

Duas sessões semanais em seis semanas, metade das sessões foram de 1 h com o psicólogo e 45min de atividades educativas com o médico e psicólogo e a outra metade de $1 \mathrm{~h}$ com 9 o psicólogo e 45min com o fisioterapeuta. As atividades desenvolvidas foram educacionais, TCC e treinamento físico progressivo. 0 grupo controle e 0 grupo experimental receberam tratamento farmacológico padrão.
Melhora da QV, do nível de dor, da capacidade física, da ansiedade, da depressão e das estratégias de enfrentamento da dor até 12 meses após a intervenção. $O$ grupo interveção relatou maior satisfação com o tratamento. $\mathrm{O}$ tratamento interdisciplinar produziu melhores resultados quando comparado à farmacoterapia padrão.
Médico (reumatologista e fisiatra), enfermeiro, fisioterapeuta e terapeuta 10 ocupacional.
Uma sessão de atividade educativa em grupo a respeito da doença de 1,5 he duração; uma sessão interativa em grupo de $2 h$ de duração envolvendo autogerenciamento focadas em TCC; uma sessão em grupo de $1 \mathrm{~h}$ de terapia física e ocupacional.
Foram associados ao aumento da chance de resposta positiva os pacientes mais jovens, mais anos de escolaridade (graduação ou pós-graduação), maior escore de depressão basal, menor contagem de tender point e ausência de histórico de abuso .

O programa foi classificado positivamente pelos participantes, no qual obtiveram melhora modesta em seus sintomas ao longo de um período de 2 anos
Médico, enfermeiro, fisioterapeuta e terapeuta ocupacional.
Clínica de fibromialgia de meio dia com consultas semanais. Foi realizada consulta clínica, seguido de avaliação conjuntamente dos demais profissionais. Sessão de 30min de fisioterapia e terapia ocupacional com atividades de discussão e educação do 
caso; Vídeo educacional de 60 min no período de espera entre especialistas; Uma sessão de atividade educativa interativa a respeito da síndrome de $1 \mathrm{~h}$ com enfermeira ou um reumatologista.

Médico (reumatologista e fisiatra), enfermeiro, fisioterapeuta e terapeuta ocupacional.
Uma sessão de atividade educativa em grupo a respeito da doença de $1,5 \mathrm{~h}$ de duração; uma sessão interativa em grupo de $2 \mathrm{~h}$ de duração envolvendo autogerenciamento focadas em TCC; uma sessão em grupo de $1 \mathrm{~h}$ de terapia física e ocupacional.
Melhora de todos indicadores da QV (menos percepção geral de saúde) e redução dos indicadores do impacto da fibromialgia (menos depressão) em 6 a 12 meses após a intervenção.

Quadro 2 - Artigos incluídos na revisão integrativa segundo atores envolvidos na ação interdisciplinar, métodos interdisciplinares empregados na pesquisa e principais achadas dos estudos. QV = qualidade de vida; QVRS= qualidade de vida relacionada à saúde; TCC= Terapia cognitivo comportamental; $A V D=$ atividades de vida diária.

\section{DISCUSSÃO}

A abordagem sobre a recuperação de pacientes com fibromialgia é uma questão desafiadora devido aos múltiplos sintomas envolvidos na síndrome, e a manifestação (intensidade, a presença ou ausência) de determinado sintoma aumenta a complexidade para tratamento dessa síndrome. Consequentemente, isso requer ações de diferentes cuidados e conhecimentos do saber para compreensão da individualidade do caso para gerar resultados satisfatórios. Isto posto, é necessário o emprego de uma abordagem ampla, como a atuação de equipes interdisciplinares de saúde, para possibilitar melhor compreensão e integralização da condição de vida do paciente ${ }^{23}$.

A diversidade de interpretações da definição de interdisciplinaridade, em razão da complexidade do tema, pode gerar diferentes formas de manejo na prática profissional das equipes de saúde ${ }^{24}$. De modo que foi constatado na presente revisão, a conceituação incoerente em quase a totalidade dos artigos analisados, sendo observado ações com características multidisciplinares e não interdisciplinares. O programa desenvolvido no Canadá denominado Program d'Appprentissage de StratégieS de Auto-Gestion Efficaces (Programa de Treinamento de Estratégias Eficientes de Autogestão) apresentado no estudo de Bourgault et al. ${ }^{16}$ no ano de 2015, exemplifica de maneira clara como seria a atuação interdisciplinar no tratamento de pacientes com fibromialgia. Esta intervenção propôs o autogerenciamento do paciente por meio da combinação do uso de técnicas educacionais, psicológicas e de exercícios físicos em que a equipe profissional envolvida atuou de maneira facilitadora e conjunta nas sessões do tratamento. O que resultou em benefícios aos pacientes ao promover a redução da percepção do nível de dor, o aumento do funcionamento, da qualidade de 
vida e da impressão de controle da dor, além de contribuir na aproximação entre os profissionais e pacientes, quando comparado com grupo que recebeu somente tratamento medicamentoso.

A proposta de programas de reabilitação de dor é essencialmente focada no autogerenciamento e autocontrole dos sintomas, com intuito de proporcionar melhores condições de vida para o cotidiano dos acometidos ${ }^{14}$. Por não haver estabelecimento internacionalmente da definição do tratamento de ações multiprofissionais no tratamento da fibromialgia ${ }^{12}$, os protocolos de intervenção dos estudos observados apresentaram diferentes estruturas (duração, frequência e quantidade das sessões). Contudo, é observado que as atividades envolvidas nos programas eram relacionadas às ferramentas da área de psicologia, a utilização de educação em saúde e a orientação para a realização de exercício físico.

Conforme mencionado nos procedimentos metodológicos da presente pesquisa, os estudos foram distribuídos em três grupamentos, sendo estes "A, B e C ", nos quais estão organizados de acordo com o objetivo de cada artigo mencionado no Quadro 01 e os principais resultados do Quadro 02.

No grupo A, dez estudos foram verificados que de alguma forma a abordagem interdisciplinar foi eficaz, sendo avaliados pelo nível da dor, por indicadores da qualidade de vida, avaliações da capacidade funcional e da motivação. Quando comparado o uso da abordagem medicamentosa padrão, a intervenção interdisciplinar alcançou resultados superiores. No estudo de Miranda et al. ${ }^{13}$ (2016), foi evidenciado que o grupo de terapia comunitária integrativa gerou custo financeiro reduzido para saúde pública. Também foi evidenciado que após a intervenção de um programa interdisciplinar de duas semanas, houve a implementação de atividades adicionais de adaptação (terapia ocupacional) ou exercício físico, estes programas adicionais acrescentaram melhorias nos resultados independe da alocação. Todavia, o programa de adaptação apresentou valores superiores e pode ser mais adequado pois foi considerado uma abordagem mais fácil e de melhor tolerância, em decorrência, ocorreu melhor adesão nesta atividade adicional ${ }^{11}$.

No grupo $B$, estão agrupados os estudos que tiveram a intenção de identificar as características que poderiam estar relacionadas com efeitos benéficos produzidos por meio das intervenções interdisciplinares. Três estudos apontaram as características que poderiam estar relacionados com a responsividade ao tratamento. Foi apresentado em uma análise longitudinal que o estado civil, número de doenças concomitantes, os anos de convívio com a dor e os níveis de ansiedade podem ter relação com a melhora após uma intervenção interdisciplinar de seis semanas, sendo evidenciado que os pacientes casados, a presença de outras comorbidades, o início do tratamento precoce e o menor nível de ansiedade tiveram relação com as níveis mais elevados de melhora da QVRS ${ }^{12}$.

Já Amris et al. ${ }^{14}$ apontaram que os pacientes que tiveram baixa ingestão basal de analgésicos como, por exemplo, acetaminofeno, maiores níveis na avaliação da dor e do Pain Detect Questionnaire podem obter maior melhora na capacidade de realizar as atividades da vida diárias após ao programa interdisciplinar de reabilitação ${ }^{14}$. Corroborando com os desfechos supracitados, Oh et al. ${ }^{20}$ relataram que após um breve programa de tratamento interdisciplinar os 
pacientes mais jovens, com maiores níveis de depressão, que apresentaram mais anos de escolaridade, que tiveram algum histórico de abusos e menor contagem de tender points, apresentaram maior melhora após a intervenção, na qual foi avaliado mediante o questionário do impacto da fibromialgia. Em visto disso, são observados diversos componentes que podem estar associados a uma melhor resposta ao tratamento interdisciplinar, e que de modo geral, podem estão relacionados com a agravamento e a presença de dor, a preconização do tratamento e o apoio social.

No grupo C, três estudos verificaram a eficácia do tratamento em determinado período de tempo pós-intervenção (curto e longo prazo) com o intuito de analisar a prolongamento do efeito adquirido após a participação ao tratamento. No estudo de Martín et al. ${ }^{18}$, as estratégias de enfrentamento da dor foram eficazes até 12 meses após a intervenção. No estudo de Jones et al..$^{21}$ (2011) houve melhora modesta nos sintomas ao longo de um período de dois anos. Por fim, o estudo de Oh et al. ${ }^{22}$ em 6 a 12 meses após a intervenção ao um breve programa de tratamento interdisciplinar, ocorreu a redução nos indicadores do impacto da fibromialgia. Diante disto, nota-se que tratamentos interdisciplinares são capazes em promover resultados positivos duradouros após sua aplicação, assim gerando benefícios contínuos na vida dos pacientes.

\section{CONSIDERAÇÕES FINAIS}

As evidências dos estudos selecionados relataram que as abordagens interdisciplinares na fibromialgia têm efeitos benéficos aos pacientes avaliados a curto e a longo prazo, nos quais estavam relacionados na melhora de indicadores da QV e no nível de dor, no aumento da capacidade funcional e motivação, na melhora do controle do sono, da ansiedade e da depressão. Além disso, três pesquisas apontaram os preditores que estariam atrelados à resposta positiva ao tratamento interdisciplinar.

Os profissionais integrantes das equipes dos programas de tratamento relatados nos estudos eram médicos reumatologistas e fisiatras, psicólogos, enfermeiros, terapeutas ocupacionais, fisioterapeutas, profissionais de educação física, nutricionistas, fonoaudiólogos e mestrando de enfermagem. Ainda, percebeu-se a escassez de estudos interdisciplinares e a conceituação equivocada sobre o entendimento do conceito de interdisciplinaridade e multidisciplinariedade.

Por fim, o objetivo da revisão foi identificar as evidências sobre a atuação das equipes interdisciplinares no atendimento de pacientes com fibromialgia, sendo observado melhorias mais expressivas ao paciente após a participação nas intervenções dos estudos encontrados na literatura. Desta forma, novos estudos com abordagem interdisciplinar para o cuidado de pacientes com fibromialgia são viáveis e necessários. 


\section{REFERÊNCIAS}

1. Heymann RE, Paiva ES, Martinez JE, Helfenstein M Jr, Rezende MC, Provenza JR, et al. Novas diretrizes para o diagnóstico da fibromialgia. Rev. Bras. Reumatol. 2017; 57(Supl. 2): S467-S76.

2. Marques AP, Espírito Santo AS, Berssaneti AA, Matsutani LA, Yuan SLK. A prevalência de fibromialgia: atualização da revisão de literatura. Rev. Bras. Reumatol. 2017; 57(4): 356-63.

3. Helfenstein M Jr, Goldenfum MA, Siena CAF. Fibromialgia: aspectos clínicos e ocupacionais. Rev Assoc Med Bras. 2012; 58(3): 358-365.

4. Oliveira LHS; Mattos RS, Castro JBP, Therezinha LUZ M. Práticas corporais de saúde para pacientes com fibromialgia: acolhimento e humanização. Physis. 2017; 27(4): 1309-32.

5. Heymann RE, Paiva ES, Helfenstein M Jr, Pollak DF, Martinez JE, Provenza JR, et al. Consenso brasileiro do tratamento da fibromialgia. Rev. Bras. Reumatol. 2010; 50: 56-66.

6. Macfarlane GJ, Kronisch C, Dean LE, Atzeni F, Häuser W, Fluß E, et al. EULAR revised recommendations for the management of fibromyalgia. Ann Rheum Dis. 2017; 76(2): 318-28.

7. Silva MVS, Miranda GBN, Andrade MA. Sentidos atribuídos à integralidade: entre o que é preconizado e vivido na equipe multidisciplinar. Interface (Botucatu). 2017; 21(62): 589-99.

8. Costa RP. Interdisciplinaridade e equipes de saúde: concepções. Mental. 2007; 5(8): 107-24.

9. Scherer MDA, Pires DEP, Jean R. A construção da interdisciplinaridade no trabalho da Equipe de Saúde da Família. Ciênc. saúde coletiva. 2013; 18(11): 3203-12.

10. Mendes KDS, Silveira RCCP, Galvão CM. Revisão integrativa: método de pesquisa para a incorporação de evidências na saúde e na enfermagem. Texto Contexto Enferm. 2008; 17(4):758-64. 
11. Bülow C, Amris K, Bandak E, Danneskiold-Samsøe B, Wæhrens EE. Improving activities of daily living ability in women with fibromyalgia: An exploratory, quasi-randomized, phase-two study, IMPROvE trial. J Rehabil Med. 2017; 49(3): $241-50$.

12. Martín J, Torre F, Aguirre U, Padierna A, Matellanes B, Quintana JM. Assessment of predictors of the impact of fibromyalgia on health-related quality of life 12 months after the end of an interdisciplinary treatment. J Affect Disord. 2017; 208: 76-81.

13. Miranda NACG, Berardinelli LMM, Sabóia VM, Brito IS, Santos RS. Práxis interdisciplinar de cuidado em grupo de pessoas que vivem com fibromialgia. Rev Bras Enferm. 2016; 69(6): 1115-23.

14. Amris K, Luta G, Christensen R, Danneskiold-Samsøe B, Bliddal H; Wæhrens EE. Predictors of improvement in observed functional ability in patients with fibromyalgia as an outcome of rehabilitation. J Rehabil Med. 2016; 48(1): 65-71.

15. Saral I, Sindel D, Esmaeilzadeh S, Sertel-Berk HO, Oral A. The effects of long- and short-term interdisciplinary treatment approaches in women with fibromyalgia: a randomized controlled trial. Rheumatol Int. 2016; 36(10): $1379-89$.

16. Bourgault P, Lacasse A, Marchand S, Courtemanche-Harel R, Charest J, Gaumond I, et al. Multicomponent interdisciplinary group intervention for self-management of fibromyalgia: a mixed-methods randomized controlled trial. PLoS One. 2015; 10(5): e0126324.

17. Martins MR, Gritti CC, Santos Jr R, Araújo MC, Dias LC, Foss MH, et al. Randomized controlled trial of a therapeutic intervention group in patients with fibromyalgia syndrome. Rev Bras Reumatol. 2014; 54(3):179-84.

18. Martín J, Torre F, Padierna A, Aguirre U, González N, Matellanes B, et al. Interdisciplinary treatment of patients with fibromyalgia: improvement of their healthrelated quality of life. Pain Pract. 2014; 14(8):721-31.

19. Martín J, Torre F, Padierna A, Aguirre U, González N, García S, et al. Six-and 12-month follow-up of an interdisciplinary fibromyalgia treatment programme: results of a randomised trial. Clin Exp Rheumatol. 2012; 30(6 Suppl 74):103-11. 
20. Oh TH, Hoskin TL, Luedtke CA, Weingarten TN, Vincent A, Kim CH, et al. Predictors of clinical outcome in fibromyalgia after a brief interdisciplinar fibromyalgia treatment program: single center experience. PM R. 2012; 4(4):257-63.

21. Jones KD, Bennett RM, Ward RL, Deodhar AA. Description of a half-day interprofessional fibromyalgia clinic with an evaluation of patient satisfaction. Am J Phys Med Rehabil. 2011; 90(10):825-33.

22. Oh TH, Stueve MH, Hoskin TL, Luedtke CA, Vincent A, Moder KG, Thompson JM. Brief interdisciplinary treatment program for fibromyalgia: six to twelve months outcome. Am J Phys Med Rehabil. 2010; 89(2):115-24.

23. Fontoura LF, Wietzke M, Moreira IJB, Garcia EL, Krug SBF. Interdisciplinaridade (além da multidisciplinaridade): em busca da integralidade através do trabalho em grupo nas ações de educação em saúde. Rev UNIABEU. 2014; $7(15): 66-75$.

24. Loch-Neckel G, Seemann G, Eidt HB, Rabuske MM, Crepaldi MA. Desafios para a ação interdisciplinar na atenção básica: implicações relativas à composição das equipes de saúde da família. Cien Saude Colet. 2009; 14(Supl. 1):1463-72. 